\title{
Prepare first, screen if necessary
}

\author{
Seeking Sickness: Medical Screening \\ and the Misguided Hunt for Disease \\ Alan Cassels \\ Greystone Books; 2012
}

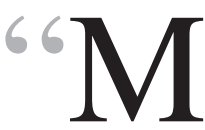
any people may take comfort in screening, in feeling they are 'doing what they can' to prevent disease," writes Alan Cassels in Seeking Sickness. "Certainly the corporate advertising, the disease groups, and the many professional organizations pump that message, implicitly saying that if you wait for symptoms to manifest, you're an idiot." In this book, Cassels, a pharma watchdog whose other titles include The ABCs of Disease Mongering (2007) and Selling Sickness (2006), pushes the opposite message: that mammograms, PSA tests, cholesterol checks, colonoscopies and even pen-and-paper mental health assessments all have unseen risks. "False positives. False negatives. Overdiagnosis. Downstream effects. Worry. Anxiety. Depression," are the

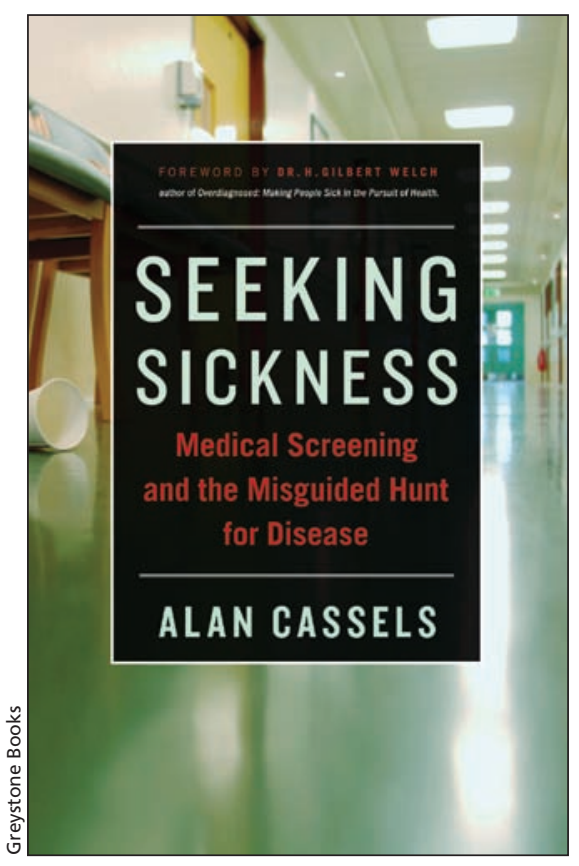

nosed (2012) and Should I Be Tested for Cancer? Maybe Not and Here's Why (2006) and Norton Hadler's Worried Sick (2008) - this small text is a good addition with compelling vignettes and a clear take-home mes-

\section{Mammograms, PSA tests, cholesterol checks, colonoscopies and even pen-and-paper mental health assessments all have unseen risks}

hazards that make the author's top-ten list as he sits in his optometrist's office, deciding whether to undergo the classic screening test for glaucoma - a puff of air to the eyes.

Though not a new claim - an array of books have called attention to the downsides of screening tests including Robert Aronowitz's Unnatural History: Breast Cancer and American Society (2007), H. Gilbert Welch's Overdiag- sage: screening tests are intended to prevent a problem, but may cause one instead, so ask a lot of questions and study up on a test before taking it.

One chapter tells the story of a USbased radiologist who had a "virtual colonoscopy" via computed tomography (CT) scan which led to his having major surgery - a thoracotomy, when the CT showed suspicious-looking lung lesions. The lesions turned out to be benign; the surgery, unnecessary. Those events took place more than a decade ago and may have reflected the radiologist's professional status, but the story serves as a segue to a clinical trial of CT colonography published in 2008. "Virtual colonoscopy a less invasive cancer screener. Scanner images replace colonoscope, lessen patient risk," ran the headline about the trial in an Atlanta newspaper article, yet an independent assessment gave the evidence a low grade and found it "insufficient" for assessing the risks and benefits of CT colonography as a screen for colon cancer.

To avoid unnecessary surgeries and other hazards, Seeking Sickness recommends quick and dirty research of the sort that could involve phoning a sibling and going to Google. Patients are told to figure out if their individual circumstances put them at increased risk for a problem before being screened for it, and find out if the screening test is endorsed by an independent body such as the US Preventive Services Task Force, or the Canadian Task Force on Preventive Health Care. This sounds simple, but may not be feasible. Entering "CT colonography" into the search engine on the website of the US Preventive Services Task Force, results in seven documents, none of which say whether to have the test.

The book's better advice is to talk to the doctor, and it offers a script of questions. As for the author, after getting his questions answered by the optometrist he had the glaucoma test and submitted calmly to the remainder of his eye exam.

\section{Miriam Shuchman MD \\ Psychiatrist \\ Toronto, Ont.}

CMAJ 2013. DOI:10.1503/cmaj.121960 